\title{
Article \\ Effect of Loading Direction on the Tensile Properties and Texture Evolution of AZ31 Magnesium Alloy
}

\author{
Yuyu Li ${ }^{1}$, Tingzhuang Han ${ }^{2}$, Zhibing Chu ${ }^{1, *}$, Chun Xue ${ }^{1}$, Qianhua Yang ${ }^{1}$, Xiaodong Zhao ${ }^{1}$ and Hong Gao ${ }^{3}$ \\ 1 College of Material Science and Engineering, Taiyuan University of Science and Technology, \\ Taiyuan 030024, China; lyy15735717698@sina.com (Y.L.); xc15735717698@sina.com (C.X.); \\ yqh15735717698@sina.com (Q.Y.); zxd15735717698@sina.com (X.Z.) \\ 2 College of Material Science and Engineering, Northwestern Polytechnical University, Xi'an 710060, China; \\ htz15735717698@sina.com \\ 3 Jiangsu Wujin Stainless Steel Co., Ltd., Changzhou 213100, China; gh15735717698@sina.com \\ * Correspondence: S20190354@stu.tyust.edu.cn; Tel.: +86-157-3571-7698
}

Citation: Li, Y.; Han, T.; Chu, Z.; Xue, C.; Yang, Q.; Zhao, X.; Gao, H. Effect of Loading Direction on the Tensile Properties and Texture Evolution of AZ31 Magnesium Alloy. Crystals 2021, 11,1193. https://doi.org/10.3390/ cryst11101193

Academic Editors: Hongbin Bei and Sergio Brutti

Received: 10 September 2021

Accepted: 28 September 2021

Published: 30 September 2021

Publisher's Note: MDPI stays neutral with regard to jurisdictional claims in published maps and institutional affiliations.

Copyright: (c) 2021 by the authors. Licensee MDPI, Basel, Switzerland. This article is an open access article distributed under the terms and conditions of the Creative Commons Attribution (CC BY) license (https:// creativecommons.org/licenses/by/ $4.0 /)$.

\begin{abstract}
Samples were cut from an extruded AZ31 magnesium alloy bar for uniaxial tensile and EBSD characterization tests. The long axis and bar extrusion directions were $0^{\circ}$ (T0 sample), $45^{\circ}$ (T45 sample), and $90^{\circ}$ (T90 sample). The effects of loading direction on the tensile behavior, microstructure, and texture evolution of the magnesium alloy were studied. Results show that the obvious mechanical anisotropy of tensile behavior is affected by the loading direction, and the T0 sample with a grain c-axis perpendicular to the extrusion direction has a strong basal texture and high flow stress and yield strength. The loading direction has a significant influence on the microstructure characteristics of different samples, especially the number of $\{10-12\}$ tensile twins and $\{10-11\}$ compression twins. Texture evolution results show that the loading direction and the effect of deformation mode on the deformation mechanism lead to variations in texture evolution: the basal slip and prismatic slip during the plastic deformation of the T0 specimen, the compression twin of the T45 specimen, and the tensile twin of the T90 specimen.
\end{abstract}

Keywords: AZ31 magnesium alloy; loading direction; tensile behavior; microstructure; texture evolution

\section{Introduction}

Owing to the advantages of magnesium alloys with high specific strength, high specific stiffness, and low densities [1,2], wrought magnesium alloys have great potential in various structural applications, particularly in the automotive, communications, and military fields [3-5]. However, the inherent close-packed hexagonal structures of magnesium alloys usually result in strong basal texture, poor ductility, and formability, which severely limits its wide-range commercial applicability [6].

Texture greatly influences the ductility and formability of magnesium alloys [7] and can be improved by reducing the strength of the base texture. Zhang et al. [8] used a one-way bending (RUB) process to improve the texture of an AZ31 magnesium alloy sheet and found that the RUB-treated sheet had a weaker base texture, which was comparable to a sheet with a strong base texture, and it had enhanced ductility. Liu et al. [9] weakened the basal structure of a magnesium alloy sheet by subjecting it through multiple passes of hot rolling, which further improved the formability of cold rolling. Iwanaga et al. [10] pointed out that reducing the (0002) base surface texture can significantly improve the room temperature formability of AZ31 sheets. Therefore, initial texture plays an essential role in the plastic deformation of magnesium alloys. However, Wang [11] pointed out that the main deformation mechanism of magnesium alloys is highly dependent on the initial texture, that is, the activation of $\{10-12\}$ tensile twins when compressed perpendicular to the c-axis or stretched parallel to the c-axis. Then, the difference in initial texture caused 
by the loading direction will inevitably affect twin deformation [12-15], and the sudden change in crystal orientation caused by the twin will render the texture evolution law complicated [16-18]. In previous studies, only magnesium alloys with a single texture shape were used in investigating microstructure evolution during deformation $[19,20]$, and the effects of different loading directions on tensile behavior, microstructure, and texture evolution were not considered. Magnesium alloys have strong anisotropy, and different deformation mechanisms will produce different deformation storage energies. Therefore, the different sampling angles of magnesium alloy extruded bars will affect the tensile deformation behavior. At present, there is no in-depth study on this issue. Therefore, the influence of loading direction on the tensile behavior and microstructure evolution during the stretching process should be explored, especially the influence of loading direction combined with deformation mechanism on texture evolution.

In this paper, from the extruded AZ31 magnesium alloy bar, the T0, T45, and T90 specimens with long axes showing large differences in the loading direction at $0^{\circ}, 45^{\circ}$, and $90^{\circ}$ from the extrusion direction were cut; then, the specimens were singled. For axis tensile analysis, through tensile test and EBSD characterization, the mechanical properties and microstructure evolution of the specimen during the tensile process were studied, and the influence of the loading direction on the tensile process was demonstrated. The viscoplastic self-consistent (VPSC) model was used in simulating and predicting the texture evolution law of tensile behavior, and the microstructure evolution mechanism of AZ31 magnesium alloy was mastered. This is of great significance for obtaining high-performance magnesium alloy materials.

\section{Viscoplastic Self-Consistent Model}

The VPSC model considers the interaction among crystal grains in a polycrystal. The model assumes that crystal grains are ellipsoidal inclusions deeply buried in an infinite homogeneous medium (homogeneous equivalent medium, HEM). The model uses a ratedependent continuous constitutive model [21] equation:

$$
\varepsilon_{i j}(\bar{x})=\sum_{s} m_{i j}^{s} \gamma^{s}(\bar{x})=\gamma_{0} \sum_{s} m_{i j}^{s}\left\{\frac{m_{k l}^{s} \sigma_{k l}(\bar{x})}{\tau^{s}}\right\}^{n}=M_{i j k l} \sigma_{k l}(\bar{x}) .
$$

In the above formula, $\tau^{s}$ is the critical shear stress; $m_{\mathrm{ij}}^{s}=\frac{1}{2}\left(n_{i}^{s} b_{j}^{s}+b_{j}^{s} n_{i}^{s}\right)$ is the Schmidt factor of the slip system/twin system (s), where $n^{s}$ and $b^{s}$ are the normal direction and slip direction/twin direction, respectively; $\varepsilon_{i j}(\bar{x})$ and $\sigma_{k l}(\bar{x})$ are the strain rate deflection tensor and stress deflection tensor, respectively; s represents each slip and twinning mechanism; $\gamma^{s}$ is the local shear rate acting on the slip system (s); $\gamma_{0}$ is the normalization coefficient; $n$ is the rate-sensitive index; and $M_{i j k l}$ is the viscoplastic convention.

The critical shear stress $\tau^{s}$ is formed with the accumulation of shear in each grain. Thus, in terms of its hardening law, it can be expressed by the empirical formula of the Voce hardening law:

$$
\tau^{\mathcal{S}}=\tau_{0}^{\varsigma}+\left(\tau_{1}^{s}+\theta_{1}^{s} \Gamma\right)\left(1-\exp \left(-\frac{\theta_{0}^{s} \Gamma}{\tau_{1}^{s}}\right)\right) .
$$

In the above formula, $\Gamma=\sum_{s} \Delta \gamma^{s}$ is the cumulative shear of the grains, and the parameters $\tau_{0}, \theta_{0}, \theta_{1}$, and $\tau_{0}^{s}+\tau_{1}^{s}$ represent the initial critical shear stress, initial hardening rate, hardening rate represented by the asymptote, and estimated critical shear stress. The specific meaning of each hardening parameter is shown in Figure 1. 


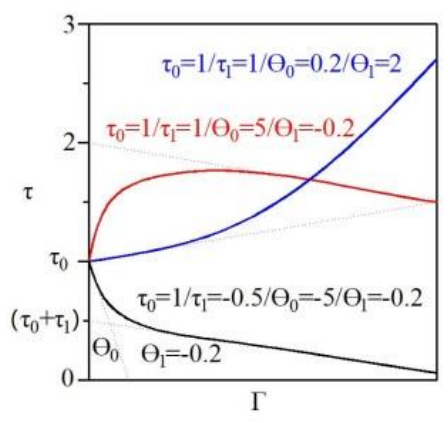

Figure 1. Physical meaning of hardening parameters.

The predominant twinning reorientation model is used for the influence of twinning on texture evolution [22]. In polycrystals, the cumulative twin crystal integral number $V^{\text {acc,mode }}$ of all twin systems in all crystal grains is:

$$
V^{\text {acc, mode }}=\sum_{g} \sum_{t} \gamma^{t, g} / S^{t}
$$

Among them, $\gamma^{t, g}$ is the shear strain caused by each twin system $t$ for a given crystal grain $g$, and the corresponding twin volume fraction is $V^{t, g}=\gamma^{t, g} / S^{t}$, where $S^{t}$ is the intrinsic shear strain of the twin system $t$.

\section{Experimental Method}

The material used in the experiment is a commercial extruded AZ31 magnesium alloy bar with a diameter of $40 \mathrm{~mm}$, from which tensile specimens with different initial orientations are cut. Figure $2 b$ shows the schematic diagram of the sheet tensile specimen designed in this paper. The total length of the specimen is $36 \mathrm{~mm}$, the gauge length is $15 \mathrm{~mm}$, and the cross-sectional dimension is $5 \mathrm{~mm} \times 1 \mathrm{~mm}$. The extrusion direction of the bar is indicated by ED, and the transverse direction perpendicular to the ED is indicated by TD. The samples with $0^{\circ}, 45^{\circ}$ and $90^{\circ}$ deflections along the ED direction are named T0, T45, and T90, respectively, as shown in Figure 2a. The EBSD technique is used in characterizing the microstructure and grain size statistics of the longitudinal section (ED-TD plane), as shown in Figure 2c. Most of the initial samples have equiaxed crystal structures, with an average grain size of approximately $25.5 \mu \mathrm{m}$ (Figure 2d). In addition, elongated crystal grains can be found in Figure 2c, resulting from the stretching of crystal grains along the extrusion direction during extrusion.

Figure 3 shows the initial pole figures of the (0001) planes of the samples. Figure $3 a$ shows that in the initial pole figure of the T0 sample, most AZ31 magnesium alloy bars have c-axis distribution perpendicular to the extrusion direction, that is, the base plane is parallel to the ED, which results in the initial magnesium alloy. The pole figure distribution presents a strong basal surface texture. When the T45 sample is loaded, the initial orientation of all grains is rotated $45^{\circ}$ in the ED direction, and the resulting texture is shown in Figure $3 \mathrm{~b}$. It can be seen that the two directions after rotation are the $45^{\circ}$ direction in the ED-TD plane, and the macroscopic strain rate and stress loaded along the two directions are the loading conditions of the $45^{\circ}$ direction. Similarly, the macroscopic strain rate and stress loaded along the TD direction are the loading conditions of the T90 specimen, as shown in Figure 3c. 


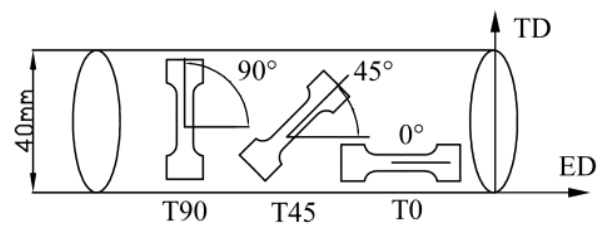

(a)

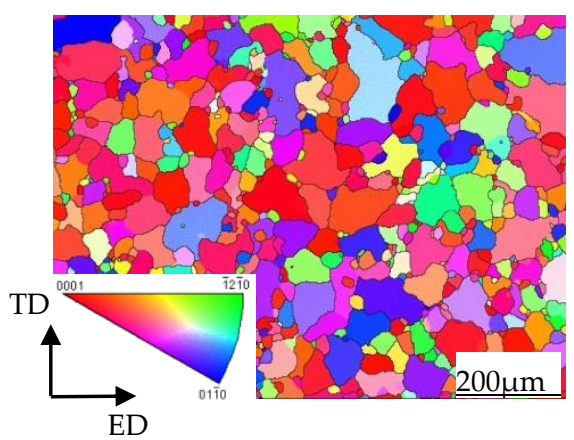

(c)

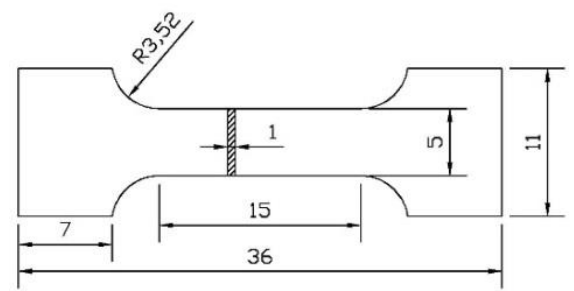

(b)

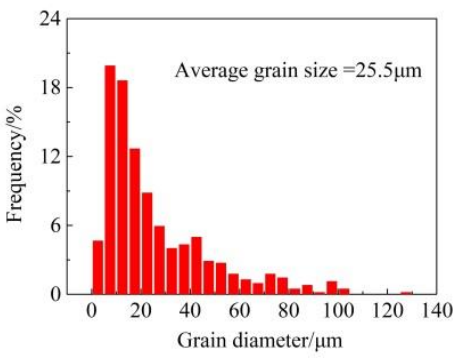

(d)

Figure 2. Specimen and microstructure: (a) sampling diagram, (b) tensile specimen, (c) microstructure, $(\mathbf{d})$ grain size.

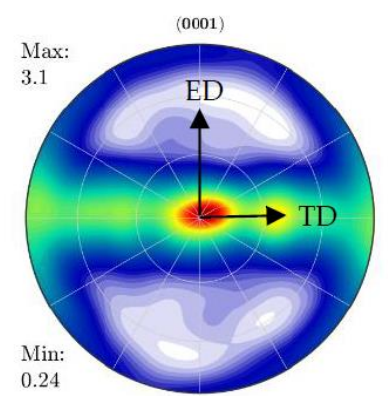

(a)

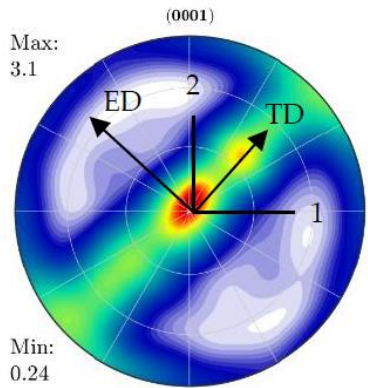

(b)

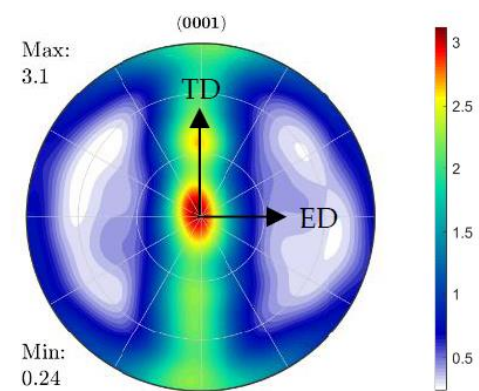

(c)

Figure 3. The initial pole figure of the $\{0001\}$ plane of different samples: (a) T0 sample, (b) T45 sample, (c) T90 sample.

The T0, T45, and T90 specimens are subjected to room temperature tensile tests. A universal material testing machine is used, and the selected strain rates are all 10-3s-1. The mechanical properties of different specimens are obtained, as shown in Figure 4 . The stress-strain curves of specimens with different loading directions have obvious mechanical anisotropy. The flow stress and yield strength of T0 specimens are significantly higher than those of the T45 and T90 specimens. 


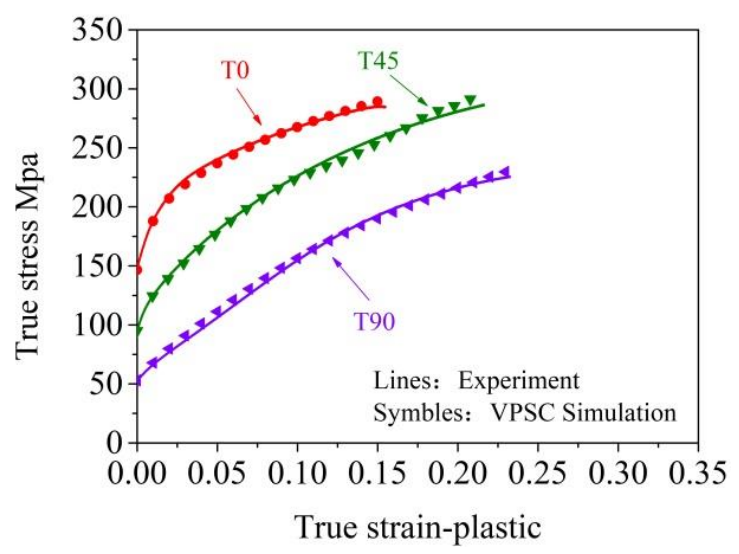

Figure 4. Fitting results of true stress-strain curves of different samples.

The VPSC model is used to fit the stress-strain curve of a specific loading experiment for the acquisition of the hardening parameters of the corresponding deformation mechanism. The specific fitting method is as follows: First, the hardening parameters of the base surface slip and prismatic surface slip are determined by fitting the tensile results of the T90 specimen, and then, the hardening parameters of the cone slip are determined by fitting the tensile results of the T45 specimen. Finally, the hardening parameters of the tensile twins and compression twins are determined by fitting the tensile results of the T0 specimens. The result of the fitting curve is shown in Figure 4. The stress-strain curve fitted by the VPSC model is in good agreement with the test results, accurately reflecting the stress at each stage of the tensile process of each specimen in different loading directions. Regarding strain characteristics, Table 1 shows the optimal hardening parameters of different deformation mechanisms obtained by fitting. At near room temperature, CRSS basal slip [16] < CRSS tensile twins [17] < CRSS prismatic slip [23] < CRSS cone slip [24] < CRSS compression twins [17]. The overall size sequence of the CRSS used in the simulation of the plastic deformation of magnesium alloys in this study is consistent with the research These parameters play important guiding roles for researchers studying the macro and micro deformation mechanisms of magnesium alloys.

Table 1. Hardening parameters of each slip and twinning system in the simulation of $\mathrm{Mg}$ alloy under monotonic loading.

\begin{tabular}{ccccc}
\hline Deformation Mode & $\boldsymbol{\tau}_{\mathbf{0}} / \mathbf{M P a}$ & $\boldsymbol{\tau}_{\mathbf{1}} / \mathbf{M P a}$ & $\boldsymbol{\theta}_{\mathbf{0}} / \mathbf{M P a}$ & $\boldsymbol{\theta}_{\mathbf{1}} / \mathbf{M P a}$ \\
\hline Basal $<\mathrm{a}>$ & 28 & 60 & 185 & 16 \\
Prismatic $<\mathrm{a}>$ & 85 & 10 & 200 & 250 \\
Pyramidal $<\mathrm{c}+$ a $>$ & 110 & 15 & 120 & 400 \\
Extension twin & 45 & 0 & 150 & 400 \\
Compression twin & 210 & 100 & 345 & 400 \\
\hline
\end{tabular}

\section{Results and Analysis}

\subsection{Deformation Mechanism Opening Amount}

Figure 5 shows the prediction results of the relative opening of the deformation mechanism during the tensile deformation of different samples. Figure 5 a shows that when the T0 sample is uniaxially stretched, the c-axis of the crystal grain is under compressive stress, and the tensile twins are difficult to open. The relative opening of all the tensile twins is extremely small (less than $1 \%$ ), and the basal surface slip and prismatic surface slip play key roles in the entire deformation process. At increased strain, the (0001) basal planes of most grains are basically parallel to the extrusion axis, and the basal surface slip is suppressed. With the continuous increase in the stress value, the slitting stress value of the non-base surface slip reaches the corresponding shear stress value, and the 
dominant deformation mechanism changes from the base surface slip to the prismatic surface <a> slip. This result is consistent with the research results of Agnew [24]. When $\varepsilon=0.02$, the tensile twins are gradually exhausted, and the activity of the compression twins begins to increase slowly. The reason is that the slip is insufficient to coordinate the c-axis compressive strain, and the generation of compression twins can coordinate the strain when the c-axis is compressed. When the T45 specimens are uniaxially stretched, the c-axes of the crystal grains are simultaneously subjected to tensile stress and compressive stress at the initial stage of deformation, and base slip, cylindrical slip, cone slip, and tensile twinning are all activated to varying degrees. As the strain increases, the dominant deformation mechanism changes from base slip to compression twinning, as shown in Figure 5b. During the uniaxial stretching of the T90 specimen, the c-axes of the crystal grains are subjected to tensile stress at the initial stage of deformation, which is conducive to the opening of the tensile twins. At increased strain, the dominant deformation mechanism changes from base slip to cone slip. In the later stage of deformation, compression twins are produced, as shown in Figure 5c.

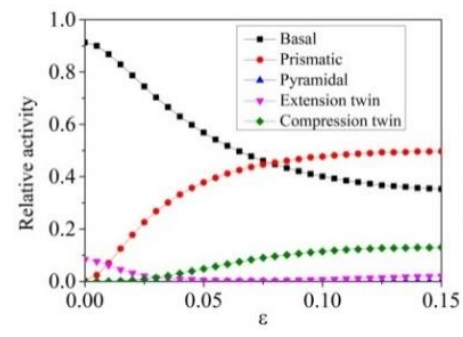

(a)

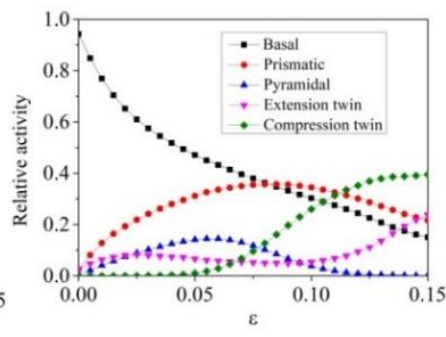

(b)

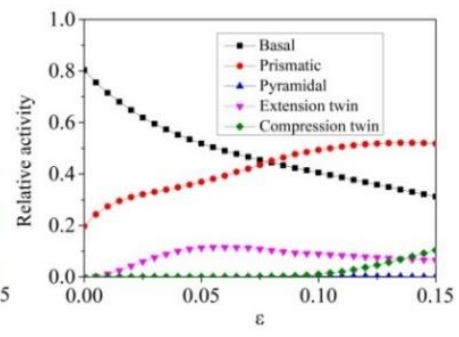

(c)

Figure 5. VPSC predicted relative activity of deformation modes under uniaxial tension of different samples: (a) T0 sample, (b) T45 sample, (c) T90 sample.

\subsection{Microstructure}

Figure 6 shows the grain orientation (IPF) diagrams of different samples when they are stretched to $8 \%$. The tensile twins and compression twins are indicated by black and white arrows, respectively. As shown in Figure 6a, a proper amount of $\{10-12\}$ tensile twins and a small amount of $\{10-11\}$ compression twins can be observed during E0 stretching. This phenomenon may be due to the inhomogeneity of the initial grain texture and orientation (the c-axis facing ED) in the initial extruded AZ31 magnesium alloy, as clearly shown in the initial pole figure (Figure 3a). Therefore, the c-axis can still be possibly pulled by the stress of the adjacent grains during the stretching process along the ED direction for the production of stretching twins. As shown in Figure 6b, when the E45 sample is stretched, a proper amount of $\{10-11\}$ compression twins and a small amount of $\{10-12\}$ tensile twins are generated. By contrast, when the E90 sample is stretched, a large amount of $\{10-12\}$ stretched twins is generated, as shown in Figure $6 c$, indicating that stretching along the $\mathrm{c}$-axis direction in the crystal grains is conducive to the generation of stretched twins.

Figure 7 shows the grain boundary diagram and the grain boundary orientation difference statistical diagram of different samples uniaxially stretched to 0.08 . The grain boundary orientation difference between $2^{\circ}$ and $5^{\circ}$ is defined as the small-angle grain boundary and is represented by the green solid line; the grain boundary orientation difference of $15^{\circ}$ or more represents the large-angle grain boundary, which is represented by the black solid line. The proportions of the small-angle grain boundaries in Figure $7 \mathrm{a}-\mathrm{c}$ are $26 \%, 22 \%$, and $14 \%$, indicating that during uniaxial stretching of AZ31 magnesium alloy, the peak value of the small-angle grain boundary gradually decreases as the loading direction changes from perpendicular to the grain c-axis to parallel to the grain c-axis mainly because of twinning. Thus, the small-angle grain boundary absorption dislocation is transformed into a large-angle grain boundary. In addition, Figure $7 \mathrm{a}, \mathrm{c}$ show that the 
grain boundary orientation difference has a peak around $80-90^{\circ}$, and the grain boundary orientation difference of Figure $7 \mathrm{~b}$ has a peak around $50-60^{\circ}$, indicating that E0 and E90 specimens produced $\{10-12\}$ tensile twins during uniaxial stretching, and E45 specimens produced $\{10-11\}$ compression twins during stretching.

Figure 8 shows the local misorientation graph (Kernel Average Misorientation, KAM) and the histogram of the local misorientation distribution when the different samples are uniaxially stretched to 0.08 . The KAM diagram is used to indicate the local dislocation density and strain degree in the microstructure. The darker the color, the greater the local misorientation [25]. The average local misorientation angles of the T0, T45, and T90 samples are $0.81,0.87$, and 0.76 , respectively. The dislocation density and strain of the T0 and T45 samples are relatively large, and the distribution is relatively uniform, whereas the dislocation density and strain of the T90 sample are low, and a large amount of dislocation density and strain are generated around the original grain boundary and the sub-grain boundary. The dislocation plugging and entanglement of the magnesium alloy increases the internal storage energy of the magnesium alloy. When the dislocations accumulate to a certain degree, the dislocations can be rearranged through slipping and climbing for them to form dislocation cells and the reduction of the internal storage energy. Dislocation cells merge to a certain size, the orientation difference with the surrounding grains exceeds at an angle of $15^{\circ}$ or greater and transforms into a large-angle grain boundary.

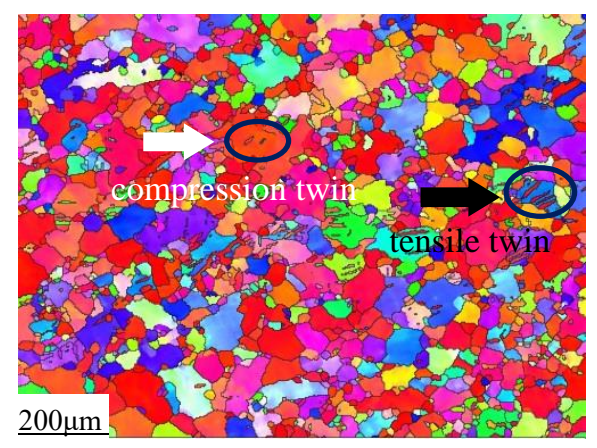

(a)

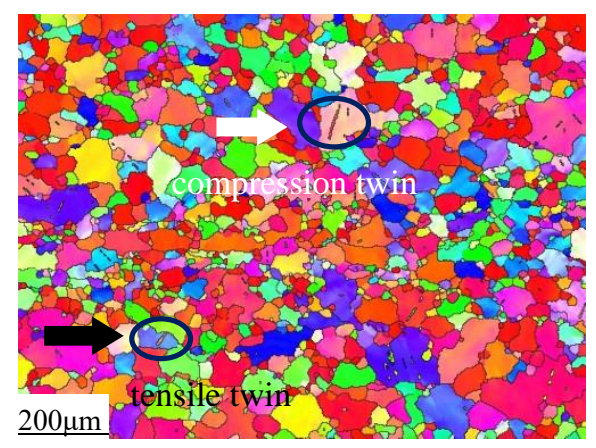

(b)

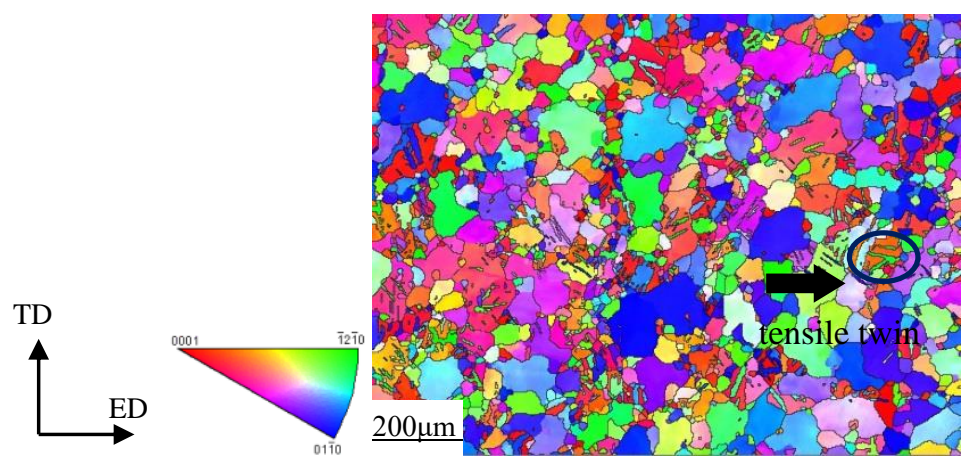

(c)

Figure 6. Grain orientation (IPF) of different samples under axial tension of 0.08: (a) T0 sample, (b) T45 sample, (c) T90 sample. 


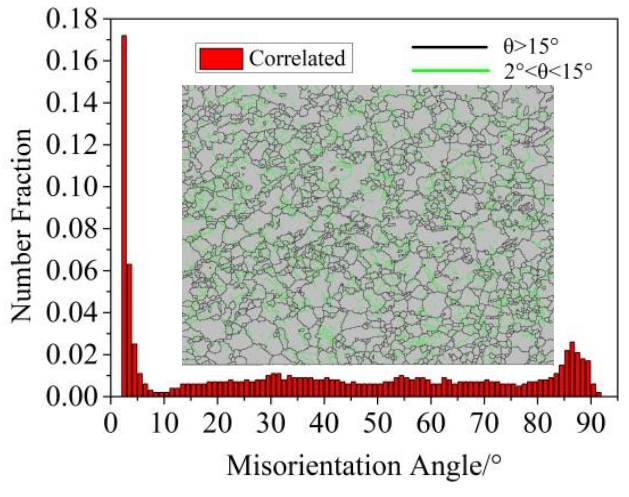

(a)

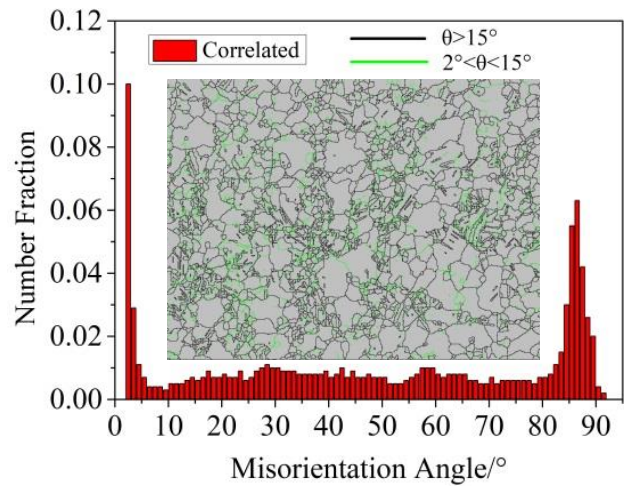

(c)

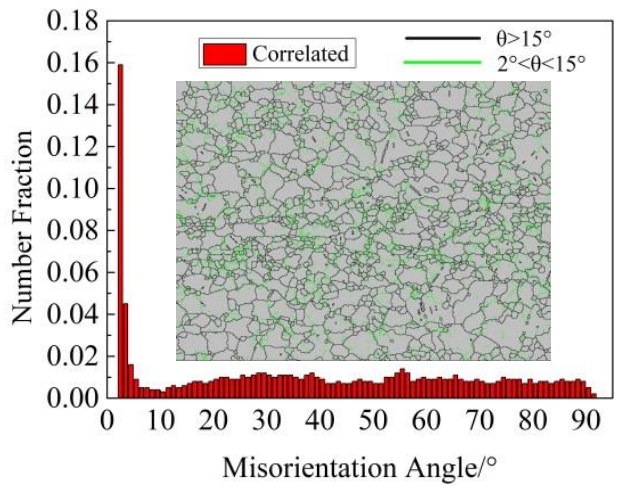

(b)

Figure 7. The grain boundary map and grain boundary misorientation map of different samples under axial tension of 0.08: (a) T0 sample, (b) T45 sample, (c) T90 sample.

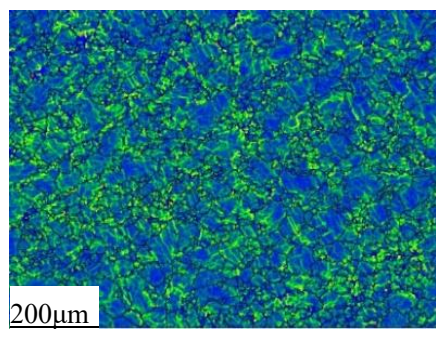

(a)

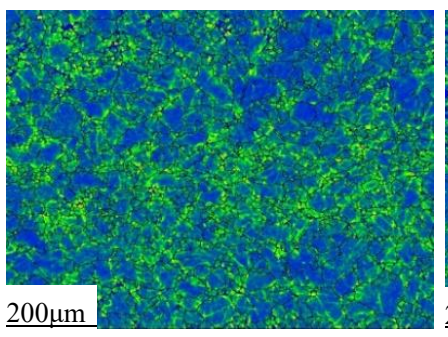

(b)

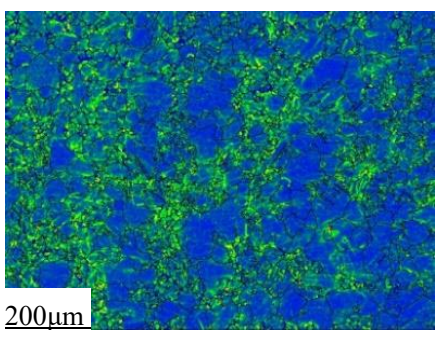

(c)

Figure 8. Local misorientation maps of different samples under axial tension of 0.08: (a) T0 sample, (b) T45 sample, (c) T90 sample.

\subsection{Texture Evolution}

Figure 9 shows the texture evolution of different samples during uniaxial stretching. When the T0 sample is stretched, the c-axes of the crystal grains show no substantial change, but the texture strength increases. When the T45 specimen is stretched, grain rotation hardly occurs at the initial stage of deformation, and the intensity peak appears near the loading direction in the (0001) pole figure at the later stage of deformation. Combined with the predicted opening rates of slip and twin systems (Figure 5), it can be seen that the $\{10-11\}$ compression twinning in the late tensile stage of T45 specimen has a certain contribution to the plastic deformation, indicating that the lattice rotation caused by the $\{10-11\}$ compression twinning is the main reason for the (0001) peak. When the T90 sample is stretched, the intensity and range of the (0001) pole figure in the TD direction are reduced, the c-axes of many crystal grains are turned to the direction perpendicular to the loading 
axis, and a certain distribution is observed in all directions along the (0001) plane. The reason is that when the T90 specimen is stretched, the loading axis is parallel or close to the direction of the c-axis of each grain. The $\{10-12\}$ tensile twinning is the dominant deformation mechanism and has a high SF. After twinning is turned on, it causes the $86.3^{\circ}$ rotation of the crystal lattice and the c-axis of the crystal grain to turn from almost parallel to the TD direction to the direction perpendicular to the TD.
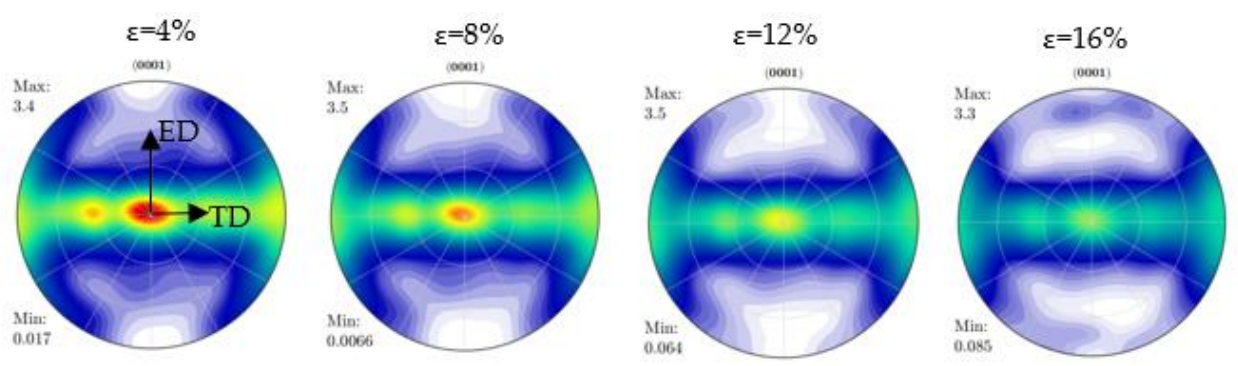

(a)
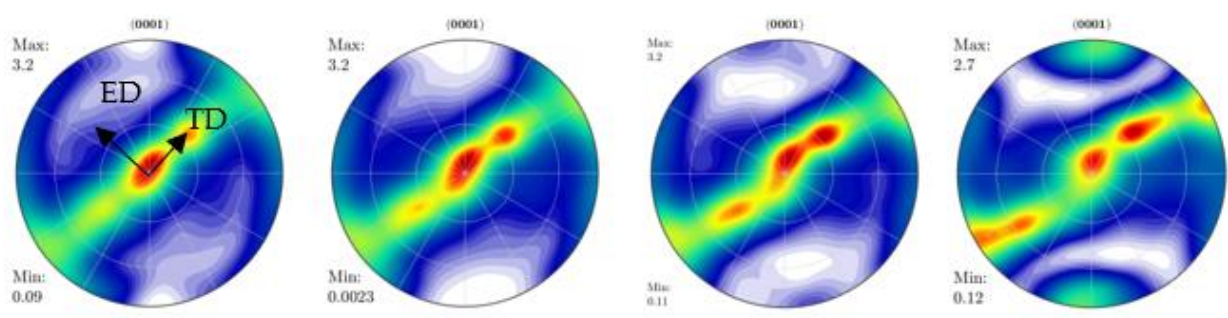

(b)
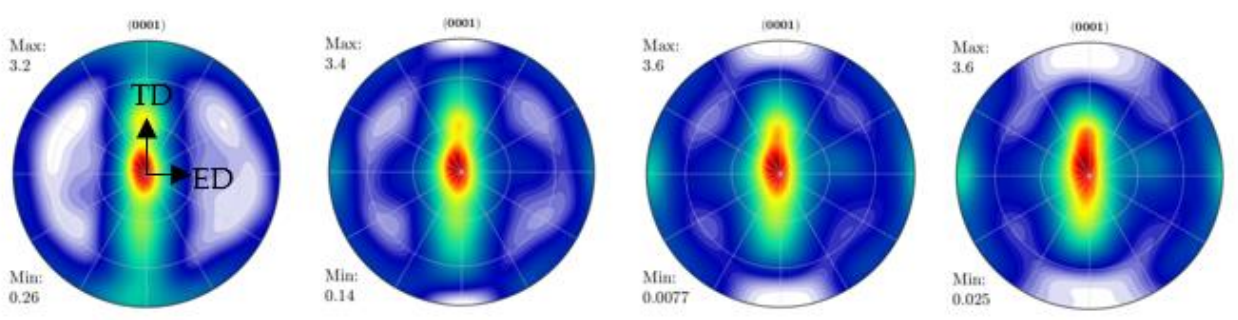

(c)

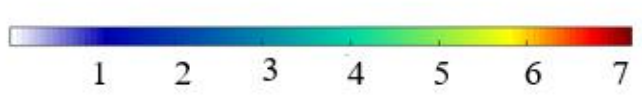

Figure 9. Predicted texture evolution of $\{0001\}$ pole figure of different samples under axial tension to different strain values: (a) T0 sample, (b) T45 sample, (c) T90 sample.

\section{Conclusions}

Through tensile tests using an EBSD characterization technology and VPSC model, the influence of loading direction on the tensile properties and texture evolution of magnesium alloys was studied, and the following conclusions were obtained:

(1) An obvious difference in the loading direction leads to the obvious anisotropy of the tensile behavior. The flow stress and yield strength of the T0 sample with a base surface texture perpendicular to the extrusion direction are significantly higher than those of the T45 and T90 samples.

(2) The microstructure results show that the reason for the large difference in the microstructure of different samples is the number of $\{10-12\}$ tensile twins and $\{10-11\}$ 
compression twins, which are mainly caused by the large difference in the loading direction.

(3) Loading direction plays an important role in the texture evolution, particularly influencing the deformation mechanism during the stretching process: owing to the base surface slip and prismatic surface slip of the T0 sample, the $\{0001\}$ pole figure is basically unchanged in the entire deformation process. In the later stage of deformation of the T45 specimen, the lattice rotation caused by the opening of the compression twins is the main reason for the peak load direction of the (0001) pole figure. The large number of tensile twins of the T90 sample is turned on, reducing the strength and range of the (0001) pole figure in the TD direction, and the c-axes of many crystal grains are turned to a direction perpendicular to the loading axis.

Author Contributions: Conceptualization, Y.L. and T.H; methodology, Y.L.; validation, C.X. and Q.Y.; formal analysis, C.X.; investigation, Q.Y.; resources, Z.C.; data curation, Y.L. and T.H.; writingoriginal draft preparation, Y.L.; writing-review and editing, X.Z. and H.G.; project administration, Z.C. All authors have read and agreed to the published version of the manuscript.

Funding: This research was funded by the National Key R\&D Program of China (2018YFB1307902), National Natural Science Foundation of China (U1710113), Joint Postgraduate Training Base of Shanxi Province (2018JD33), Top young talents in Shanxi Province, Shanxi Excellent Youth Fund (201901D211312), Innovative talents in Colleges and universities of Shanxi Province, Outstanding young academic leaders in Shanxi Province, Transformation and cultivation project of scientific and technological achievements in Colleges and universities of Shanxi Province (2019KJ028), Leading talents of emerging industries in Shanxi Province, Postgraduate Education Innovation Program of Shanxi Province (2019SY482)

Institutional Review Board Statement: Not applicable.

Informed Consent Statement: Not applicable.

Data Availability Statement: Data sharing not applicable.

Acknowledgments: The authors express their thanks to the people helping with this work, and acknowledge the valuable suggestions from the peer reviewers.

Conflicts of Interest: The authors declare no conflict of interest.

\section{References}

1. Wan, D.Q. Near spherical $\alpha$-Mg dendrite morphology and high damping of low-temperature casting Mg-1wt.\%Ca alloy. Mater. Charact. 2011, 62, 8-11.

2. Chino, Y.; Sassa, K.; Kamiya, A.; Mabuchi, M. Stretch formability at elevated temperature of a cross-rolled AZ31 Mg alloy sheet with different rolling routes. Mater. Sci. Eng. A 2008, 473, 195-200. [CrossRef]

3. Pan, H.; Qin, G.; Huang, Y.; Ren, Y.; Sha, X.; Liu, Z.; Li, C.; Wu, X.; Chen, H.; He, C.; et al. Development of low-alloyed and rare-earth-free magnesium alloys having ultra-high strength. Acta Mater. 2018, 149, 350-363. [CrossRef]

4. Zeng, Z.; Stanford, N.; Davies, C.H.J.; Nie, J.-F.; Birbilis, N. Magnesium extrusion alloys: A review of developments and prospects. Int. Mater. Rev. 2018, 64, 1-36. [CrossRef]

5. Lu, X.; Zhao, G.; Zhou, J.X.; Zhang, C. Effect of extrusion speeds on the microstructure, texture and mechanical properties of high-speed extrudable Mg-ZnSn-Mn-Ca alloy. Vacuum 2018, 157, 180-191. [CrossRef]

6. Somekawa, H.; Kinoshita, A.; Washio, K.; Kato, A. Enhancement of room temperature stretch formability via grain boundary sliding in magnesium alloy. Mater. Sci. Eng. A 2016, 676, 427-433. [CrossRef]

7. Qin, D.H.; Wang, M.J.; Sun, C.Y.; Su, Z.X.; Qian, L.Y.; Sun, Z.H. Interaction between texture evolution and dynamic recrystallization of extruded AZ80 magnesium alloy during hot deformation. Mater. Sci. Eng. A 2020, 788, 139537. [CrossRef]

8. Zhang, H.; Huang, G.; Fan, J.; Roven, H.J.; Xu, B.; Dong, H. Deep drawability and drawing behaviour of AZ31 alloy sheets with different initial texture. J. Alloys Compd. 2014, 615, 302-310. [CrossRef]

9. Liu, D.; Liu, Z.; Wang, E. Improving single pass reduction during cold rolling by controlling initial texture of AZ31 magnesium alloy sheet. Trans. Nonferrous Met. Soc. China 2018, 28, 244-250. [CrossRef]

10. Iwanaga, K.; Tashiro, H.; Okamoto, H.; Shimizu, K. Improvement of formability from room temperature to warm temperature in AZ-31 magnesium alloy. J. Mater. Process. Technol. 2004, 155-156, 1313-1316. [CrossRef]

11. Wang, Y.; Choo, H. Influence of texture on Hall-Petch relationships in an Mg alloy. Acta Mater. 2014, 81, 83-97. [CrossRef]

12. Wang, H.; Wu, P.D.; Wang, J.; Tomé, C.N. A physics-based crystal plasticity model for hexagonal close packed (HCP) crystals including both twinning and detwinning mechanisms. Int. J. Plast. 2013, 49, 36-52. [CrossRef] 
13. Wu, P.D.; Guo, X.Q.; Qiao, H.; Agnew, S.R.; Lloyd, D.J.; Embury, J.D. On the rapid hardening and exhaustion of twinning in magnesium alloy. Acta Mater. 2017, 122, 369-377. [CrossRef]

14. Balík, J.; Dobroň, P.; Chmelík, F.; Kužel, R.; Drozdenko, D.; Bohlen, J.; Letzig, D.; Lukáč, P. Modeling of thework hardening in magnesium alloy sheets. Int. J. Plast. 2015, 76, 166-185. [CrossRef]

15. Barnett, M.R.; Ghaderi, A.; Fonseca, J.Q.; Robson, J.D. Influence of orientation on twin nucleation and growth at low strains in a magnesium alloy. Acta Mater. 2014, 80, 380-391. [CrossRef]

16. Lou, X.Y.; Li, M.; Boger, R.K.; Agnew, S.R.; Wagoner, R.H. Hardening evolution of AZ31B Mg sheet. Int. J. Plast. 2007, 23, 44-86. [CrossRef]

17. Koike, J. Enhanced deformation mechanisms by anisotropic plasticity in polycrystalline $\mathrm{Mg}$ alloys at room temperature. Metall. Mater. Trans. A 2005, 36, 1689-1696. [CrossRef]

18. Huang, H.T.; Godfrey, A.; Zheng, J.P.; Liu, W. Influence of local strain on twinning behavior during compression of AZ31 magnesium alloy. Mater. Sci. Eng. A 2015, 640, 330-337. [CrossRef]

19. Kim, S.-H.; Lee, J.H.; Lee, C.S.; Yoon, J.; Park, S.H. Dynamic deformation behavior and microstructural evolution during high-speed rolling of Mg alloy having non-basal texture. J. Mater. Sci. Technol. 2019, 4, 473-482. [CrossRef]

20. Serebryanyi, V.N.; Khar'kova, M.A.; Lukyanova, E.A.; Karelin, F.R. Texture, Structure, Mechanical Properties, and Deformability of MA2-1pch Magnesium Alloy Rolled Sheets Preliminarily Subjected to Direct Extrusion or Equal-Channel Angular Pressing and Annealing. Russ. Metall. (Metally) 2018, 2018, 465-472. [CrossRef]

21. Kabirian, F.; Khan, A.S.; Gnäupel-Herlod, T. Visco-plastic modeling of mechanical responses and texture evolution in extruded AZ31 magnesium alloy for various loading conditions. Int. J. Plast. 2015, 68, 1-20. [CrossRef]

22. Agnew, S.R.; Brown, D.W.; Tomé, C.N. Validating a polycrystal model for the elastoplastic response of magnesium alloy AZ31 using in situ neutron diffraction. Acta Mater. 2006, 54, 4841-4852. [CrossRef]

23. Reed-Hill, R.E.; Robertson, W.D. Additional modes of deformation twinning in magnesium. Acta Metall. 1957, 5, 717-727. [CrossRef]

24. Agnew, S.R.; Yoo, M.H.; Tomé, C.N. Application of texture simulation to understanding mechanical behavior of Mg and solid solution alloys containing Li or Y. Acta Mater. 2001, 49, 4277-4289. [CrossRef]

25. Yang, Y.; Yang, X.; Xiao, Z.; Zhang, D.; Wang, J.; Sakai, T. Annealing behavior of a cast Mg-Gd-Y-Zr alloy with necklace fine grains developed under hot deformation. Mater. Sci. Eng. A. 2017, 688, 280-288. [CrossRef] 